\title{
INTERDISCIPLINARIDADE EM CONTABILIDADE GERENCIAL E CONTABILIDADE DE CUSTOS EM PROGRAMAS DE MESTRADO: PERCEPÇÕES DE DOCENTES E DISCENTES
}

\section{INTERDISCIPLINARITY IN MANAGEMENT ACCOUNTING AND COST ACCOUNTING IN MASTERS PROGRAMS: PERCEPTIONS OF TEACHERS AND STUDENTS}

\section{Resumo}

\author{
Claudio Marcelo Edwards Barros ${ }^{a}$; Luiz Carlos Lemos Júnior ${ }^{b}$; \\ Romualdo Douglas Colauto ${ }^{c}$; Simone Bernardes Voese ${ }^{d}$ \\ ${ }^{a}$ Gerente Financeiro de Sykes do Brasil Ltda \\ Mestre em Contabilidade pela Universidade Federal do Paraná, UFPR \\ Curitiba,PR-Brasil; - E-mail: claudiomedwards@hotmail.com \\ brofessor do Programa de Especialização em Contabilidade da Pontifícia Universidade Católica de Campina \\ Mestre em Contabilidade pela Universidade Federal do Paraná - UFPR \\ Campinas, SP-Brasil; E-mail: luiz.lemos@puc-campinas.edu.br \\ ${ }^{c}$ Professor Adjunto do Programa de Mestrado em Contabilidade da Universidade Federal do Paraná - UFPR \\ Doutor em Engenharia de Produção pela Universidade Federal de Santa Catarina, UFSC \\ Curitiba,PR-Brasil; - E-mail: rdcolauto@ufpr.br \\ ${ }^{d}$ Professora Adjunta do Programa de Mestrado em Contabilidade Universidade Federal do Paraná - UFPR \\ Doutora em Engenharia de Produção pela Universidade Federal de Santa Catarina, UFSC \\ Curitiba,PR-Brasil; - E-mail: simone.voese@gmail.com
}

O presente artigo tem como objetivo analisar a percepção de docentes e discentes sobre a prática de interdisciplinaridade nas disciplinas de contabilidade gerencial e contabilidade de custos ministradas em programas de mestrado em contabilidade. A amostra da pesquisa é composta por 5 instituições de ensino superior localizadas nos estados de Minas Gerais, Santa Catarina, Amazonas, Paraná e Rio Grande do Sul. Trata-se de uma pesquisa exploratória que utilizou perguntas fechadas como instrumento e contou com 77 respondentes de um total de 148. Para análise quantitativa de dados, utilizou-se Análise Fatorial Exploratória (AFE) visando estimar os fatores subjacentes às variáveis relacionadas aos elementos promotores de interdisciplinaridade. Os resultados indicam que a percepção de presença de interdisciplinaridade nos programas de mestrado ocorre para $51 \%$ dos respondentes, enquanto que $49 \%$ afirmaram não haver ou desconhecer a presença de práticas de interdisciplinaridade nos projetos pedagógicos dos cursos. Assim, os resultados sugerem haver a apreciação isolada do conteúdo destas duas disciplinas e reforça a concepção de que a interdisciplinaridade, no ensino da contabilidade, nem sempre é observada de forma explícita nas matrizes curriculares e nas ementas dos cursos.

Palavras-chave: Interdisciplinaridade; Contabilidade gerencial; Contabilidade de custos.

\begin{abstract}
This paper is aimed at analyzing the perception of teachers and students about the interdisciplinarity among the disciplines of management accounting and cost accounting, taught in Masters programs. The survey sample consisted of five educational institutions in the states of Minas Gerais, Santa Catarina, Amazonas, Parana and Rio Grande do Sul. This is an exploratory study that used closed questions as a research tool and had 77 respondents out of a total of 148. For the quantitative analysis of data, it was applied the Exploratory Factorial Analysis (EFA) to estimate the factors surrounding the variables related to the promoter's elements of interdisciplinary. The results indicate that the perception of the presence of interdisciplinary in Masters programs occurs for $51 \%$ of respondents, while $49 \%$ said that there was no interdisciplinary or ignore the presence of interdisciplinary practices in the pedagogical courses. Therefore, the results suggests the isolated appreciation of the content of these two disciplines and reinforces the conception that the interdisciplinary, on the teaching of accounting, is not always observed on its explicit form on its curricular matrixes and on the courses emends.
\end{abstract}

Keywords: Interdisciplinarity; managerial accounting; cost accounting. 


\section{INTRODUÇÃO}

O tema interdisciplinaridade é tido como um dos enfoques de discussões de pautas de reuniões em ambiente acadêmico, pois esse novo olhar para os conhecimentos põe em cheque outro problema: o de garantir a especificidade de cada conteúdo e sua área de atuação (MANECHINE, 2006). Santomé (1998) defende que uma educação voltada à reconstrução dos conteúdos, cujos significados possam ser articulados para compreensão de um fenômeno ou mesmo de um novo propósito de conhecimento, não deve ser exaustivamente fragmentada.

Santomé (1998) contesta a postura comumente observada do professor que aponta maior importância relativa à disciplina ministrada sob sua responsabilidade. Esta postura força o aluno a direcionar mais atenção a uma disciplina, em detrimento de outras. Assim, apontar maior importância para sua disciplina e forçar os estudantes a direcionarem mais atenção para certos conteúdos, resulta na fragmentação do conhecimento e contradiz a argumentação defendida por Germain (1991) de que a interdisciplinaridade pressupõe a existência de pelo menos duas disciplinas de referência e a presença de uma ação recíproca. Neste sentido Machado (1995, p. 186) reforça que "o significado curricular de cada disciplina não pode resultar de uma apreciação isolada de seu conteúdo, mas sim do modo como se articulam as disciplinas em seu conjunto".

Para Piaget (1972), a interdisciplinaridade constitui-se no intercâmbio e integração de várias ciências. Esta cooperação tem como resultado o enriquecimento das partes envolvidas nas relações de ensino e pesquisa. As abordagens envolvendo o ensino interdisciplinar ganharam espaço nos estudos e pesquisas voltados aos problemas ocasionados pela fragmentação do conhecimento em disciplinas no final do século XX (MORIN, 1999). Sob esta perspectiva, mas em intensidades diferentes, a pesquisa e o ensino passaram a ser vistos como processos reprodutores de conhecimento.

Os programas de mestrado em contabilidade são o lócus capacitador de profissionais iniciantes na pesquisa e na docência. Entende-se, portanto, que os discentes de programas dessa natureza devam ser orientados por professores que detenham tais competências necessárias para o exercício da docência, alinhados com a missão e visão do projeto pedagógico ao qual estão ligados. Obviamente, espera-se que dentre os atributos da docência esteja promoção da interdisciplinaridade.

Estudos sobre interdisciplinaridade no contexto do ensino da contabilidade em cursos de pós-graduação na área de Ciências Sociais Aplicada é um tema relativamente novo, embora recorrente nas pesquisas brasileiras. Parte destes trabalhos justifica seus objetivos tendo como base os desafios enfrentados pelas instituições de ensino superior em proporcionar formação diversificada a profissionais da área de Ciências Contábeis. Aliado a isto e na busca de vencer os desafios inerentes ao processo educacional, as disciplinas curriculares dos cursos de Pósgraduação em Contabilidade também necessitam de estratégias que lhes permitam desenvolver a prática da interdisciplinaridade.

Neste sentido este estudo busca responder a seguinte questão de pesquisa: qual a percepção de docentes e discentes sobre a prática de interdisciplinaridade entre as disciplinas de contabilidade gerencial e contabilidade de custos ministradas nos mestrados em contabilidade? Consequentemente, o objetivo geral do estudo consiste em analisar a percepção de docentes e discentes sobre a prática de interdisciplinaridade nas disciplinas de 
contabilidade gerencial e de custos ministradas em programas de mestrado em contabilidade. $\mathrm{O}$ estudo é importante porque os programas de pós-graduação representam o locus de formação de docentes para o ensino superior da contabilidade. Portanto, a verificação dos desdobramentos de práticas de interdisciplinaridade em programas de mestrado contribui ao debate sobre ensino e aprendizagem.

\section{REFERENCIAL TEÓRICO}

\subsection{A interdisciplinaridade no ensino da contabilidade gerencial e de custos}

A interdisciplinaridade consiste na integração de diferentes áreas do ensino adicionada a medidas de planejamento, cooperação e trocas de conhecimento entre disciplinas. Ao contrário da multidisciplinaridade e da pluridisciplinaridade, necessita haver uma coordenação que integre os objetivos, atividades, procedimentos e que propicie o intercâmbio e o diálogo (NOGUEIRA, 1998). Fazenda (1992) afirma que interdisciplinaridade pressupõe uma postura interdisciplinar representada por atitudes de busca, envolvimento e compromisso diante do conhecimento. Luck (1995) apud PASSOS (2004, p. 27) define interdisciplinaridade como processo que envolve "trabalho conjunto de integração das disciplinas do currículo escolar, entre si e com a realidade, de modo a superar a fragmentação do ensino".

Ferreira (2001) ressalta que a integração e a inter-relação dos componentes curriculares não garantem um ensino interdisciplinar. É preciso que os conhecimentos delineados nos planos pedagógicos se articulem e se organizem em uma totalidade, procurando envolver o corpo escolar numa intenção consciente. Assim, o que caracterizaria uma prática interdisciplinar seria o sentido intencional que ela carrega. Ou seja, é necessária uma atitude mental.

Zabala (2002, p. 33) explica que uma das principais formas de interdisciplinaridade é a "interação entre duas ou mais disciplinas", de forma que esta colaboração entre as ciências, com o mesmo objetivo, pudesse ser construída, rompendo as fronteiras ou limites das disciplinas. Muitas das ideias que nascem nas fronteiras e nas zonas incertas entre as disciplinas e algumas grandes descobertas ou teorias, provavelmente, nasceram de forma interdisciplinar (MORIN, 2002).

No Brasil, o projeto pedagógico do ensino da contabilidade deve abranger, sem prejuízo de outros elementos estruturais, formas de realização da interdisciplinaridade. No entanto, tais formas não são indicadas pelas regras vigentes. Krasilchik (1998) comenta que o ideário da interdisciplinaridade já se faz presente, nas agências de fomento, nas discussões sobre as políticas de incentivo a pesquisa e reformulações curriculares.

A relação pedagógica decorre do processo dinâmico e interativo (DALBEN; CASTRO, 2010) onde os participantes partilham conhecimentos mediante movimentos coordenados que culminam na aprendizagem. Para Fazenda (1992) posturas direcionadas a ações de interação e envolvimento entre docente e discente resultam em interdisciplinaridade. Interdisciplinaridade pode ser obtida mediante o diálogo das partes interessadas e por meio da criação de possibilidades de redimensionamento de métodos de ensino. Para Gusdorf (1990) o prefixo "inter" não se restringe a pluralidade ou justaposição; evoca também um espaço comum de saberes diferentes. A interdisciplinaridade supõe além de postura direcionada a este fim, curiosidade e abertura de pensamento. De acordo com Santomé (1998) a interdisciplinaridade envolve não somente 
estudo coletivo, parceria entre as disciplinas e a interdependência entre elas. Requer, sobretudo, uma postura mental e metodológica que leva a mudanças de crenças anteriores e possibilite a aquisição dos conceitos estudados. O intercâmbio entre os saberes pode se compor levando-se em consideração circunstâncias como a disponibilidade de tempo do corpo docente, confiança e aceitação de risco.

Estudos contemporâneos (ALTHEMAN, 1998; PADOAN E CLEMENTE, 2006; ALTHOFF E DOMINGUES, 2008; MORAES, ARAÚJO E ARAÚJO, 2009; BERNARDO, NASCIMENTO E NAZARETH, 2010) preocuparam-se em abordar a interdisciplinaridade e o ensino da administração e contabilidade em cursos de graduação de instituições brasileiras sob variados aspectos.

Altheman (1998) estudou a adoção de pesquisa coletiva como uma das possibilidades de monitoramento e execução de um projeto interdisciplinar no ensino de Administração em instituições brasileiras de ensino superior. Para o autor esse mecanismo permitiu notar que o professor deixa de se opor às mudanças pretendidas, representando uma solução possível ao isolamento disciplinar reforçado por Machado (1995). Ademais, a cooperação entre os professores e alunos torna-se mais notada e proveitosa.

Padoan e Clemente (2006) analisaram a percepção dos docentes de 8 instituições de ensino superior sobre a importância da interdisciplinaridade na formação do contador; constataram baixo nível de interdisciplinaridade percebida por parte dos docentes. Observaram, por exemplo, que disciplinas como estatística, economia, comportamento organizacional e sociologia são consideradas como pouco importantes para o ensino da contabilidade.

Althoff e Domingues (2008) verificaram a percepção de discentes e coordenadores de cursos de graduação de ciências contábeis em uma macrorregião na região do Vale do Itajaí no Estado de Santa Catarina. Os resultados mostram nível inferior de relacionamento entre disciplinas, o que contraria o conceito de interdisciplinaridade. As práticas observadas nas instituições pesquisadas estão associadas à pluridisciplinaridade e multidisciplinaridade e disciplinaridade cruzada, mas não a interdisciplinaridade.

Moraes, Araújo e Araújo (2009) buscaram identificar nas universidades do Rio Grande do Norte, a percepção dos discentes sobre a atitude interdisciplinar dos docentes. Os resultados da pesquisa indicaram que professores das instituições pesquisadas demonstram atitudes parciais de interdisciplinaridade. Práticas de pesquisa e de interdisciplinaridade podem estar vinculadas para um objetivo comum: contribuir para o processo de ensino e aprendizagem.

Partindo deste contexto, Bernardo, Nascimento e Nazareth (2010), objetivaramidentificar as características relacionadas à coordenação do ensino e práticas de interdisciplinaridade em 33 cursos de ciências contábeis de 38 instituições de ensino superior do Estado de Minas Gerais. Os resultados sugerem que um dos fatores limitantes para o desenvolvimento de pesquisas na área de contabilidade é a elevada inserção de discentes no mercado de trabalho. Observaram que práticas de interdisciplinaridade estão associadas ao esforço das coordenações dos cursos de graduação em promover a revisão das matrizes curriculares. Além disso, práticas de interdisciplinaridade foram observadas nas disciplinas de contabilidade internacional e controladoria.

A interdisciplinaridade no ensino da contabilidade nem sempre é observada de forma explícita nas matrizes curriculares e nas ementas dos cursos. Embora a legislação e as normas 
vigentes não façam menção às matrizes curriculares e aos projetos pedagógicos dos cursos de pós-graduação, entende-se que as disciplinas e conteúdos ofertados por esses, devem privilegiar a efetiva implementação de ações e atitudes interdisciplinares. .

Segundo Walker (2005), a história do ensino da Contabilidade revela a importância do vínculo entre as disciplinas, baseados em perspectivas interdisciplinares. Laffin (2004, p. 148) comenta que no ensino-aprendizagem em contabilidade "é fundamental que a integração das disciplinas seja realizada nos semestres ou anuidades, conforme dispõe a organização institucional, para garantir a imbricação, ao mesmo tempo em que promove a visão de totalidade do conhecimento, assim como sua pertinência".

Projetos contemporâneos mostrando coesão entre teoria e prática contábil por meio do trabalho interdisciplinar (ROSLENDER; DILLARD, 2003). Fragoso, Ribeiro Filho e Libonati (2006), afirmam que o processo de pesquisa dos doutores em Contabilidade no Brasil, evidencia o impacto da interdisciplinaridade quando se tem pelo menos uma formação seja em especialização, mestrado ou doutorado fora da área contábil. Esses pesquisadores verificaram que o perfil interdisciplinar torna-se consistente e atuante no processo de produção científica ao longo do tempo.

A definição das bases limítrofes das várias áreas da contabilidade é complexa. Contudo, as características individuais de cada área indicam seu objeto próprio de estudo e apresentam o conjunto de temáticas de concentração. No que se refere ao contexto de interdisciplinaridade no ensino da disciplina de contabilidade gerencial, contabilidade de custos e contabilidade financeira, Padoveze (2000) destaca o isolamento do ensino da disciplina de contabilidade gerencial. Este aspecto fora discutido anteriormente por Machado (1995), que ressaltou o problema ainda que não associado à área de contabilidade.

Padoveze (2000, p. 32) ressalta que no âmbito didático essas disciplinas são ensinadas isoladamente. Em função disso é comum que o aprendizado dos temas constantes em cada disciplina seja feito de forma não integrada. Isto que dizer que quando um aluno está aprendendo as técnicas de formação do custo do produto, por exemplo, ele e o professor estão exclusivamente preocupados com isso. Assim, não há preocupação de integração com as demais áreas contábeis.

O objeto da contabilidade de custos é a organização como um todo, pois atua sobre produtos, serviços, componentes operacionais e administrativos, ou seja, tudo que compõem sua estrutura funcional (Leone, 2000), buscando promover a eficiência dos processos produtivos. Atua também, para fins legais, na composição dos custos de bens como estoques e construções ou mesmo voltada para a Contabilidade Gerencial (MAUSS; SOUZA, 2008).

Martins (2008) afirma que além de sua função legal, a Contabilidade de Custos possui outras duas funções de considerável relevância:

- (a) auxílio ao controle: sua importante missão é fornecer dados para o estabelecimento de padrões, orçamentos e outras formas de previsão e, num estágio imediatamente seguinte, acompanhar o efetivamente acontecido em comparação com os valores anteriormente definidos; e

- (b) auxílio a tomada de decisão: cujo papel reveste-se de suma importância, pois diz respeito às consequências de curto, médio e longo prazos sobre diversas questões de 
ordem gerencial.

Por meio da ausência de preocupação destacada por Padoveze (2000) e das definições acima, as disciplinas de Contabilidade Gerencial e de Custos deveriam estar integradas no contexto do ensino em instituições de ensino superior. Entende-se que diante das reflexões apontadas, faz-se necessário pensar nas práticas de ensino como um movimento de relações entre os conhecimentos, de maneira que suscite a criação e o diálogo entre as áreas dos saberes, em especial da Contabilidade Gerencial e de Custos, possibilitando aos professores relacionarem suas disciplinas como parte integrante de um todo.

\section{PROCEDIMENTOS METODOLÓGICOS}

Esta é uma pesquisa de campo exploratória realizada em cinco instituições de ensino superior situadas nas regiões Sul, Sudeste e Norte do Brasil. Todas as instituições participantes da pesquisa possuem Programas de Pós Graduação de Mestrado em Contabilidade. O objetivo da pesquisa é verificar a percepção de docentes e discentes sobre a prática de interdisciplinaridade relacionada às disciplinas de contabilidade gerencial e de custos. Nesta etapa será apresentada a formação da amostra da pesquisa, características do instrumento de pesquisa utilizado, as variáveis utilizadas no estudo e o conjunto de ferramentas estatísticas para realização da análise de dados.

Foram selecionadas intencionalmente três universidades públicas federais e duas universidades privadas: Universidade Federal do Amazonas (UFAM), Universidade Federal de Minas Gerais (UFMG), a Universidade Federal do Paraná (UFPR), a Universidade do Vale do Rio Sinos (UNISINOS) e Universidade Regional de Blumenau (FURB). O motivo da escolha destas universidades está relacionado ao fato de todas contemplarem, em suas matrizes curriculares de mestrado, as disciplinas de contabilidade gerencial e contabilidade de custos.

Na Tabela 1, apresenta-se a delimitação da amostra por meio dos respondentes da pesquisa para as 5 instituições de ensino.

Tabela 1: Amostra da pesquisa

\begin{tabular}{lccc}
\hline & População & Respondentes & \% de respondentes \\
\hline Discentes & 140 & 67 & 47,85 \\
Docentes & 10 & 8 & 80,00 \\
Total & 148 & 77 & 52,03 \\
\hline
\end{tabular}

A população de estudantes matriculados no ano de 2010 é de 140, dos quais 67 responderam a pesquisa, representando $47,85 \%$. Ressalta-se que todos os docentes pesquisados são aqueles que efetivamente trabalham as disciplinas de contabilidade gerencial e de custos. Das 5 instituições pesquisadas, em 4 todos os docentes que ministram estas disciplinas responderam o questionário enviado.

A coleta de dados envolveu a aplicação de questionário com perguntas fechadas para obtenção da percepção docente e discente sobre a prática interdisciplinar, entre as disciplinas de contabilidade gerencial e de custos. O instrumento de coleta está dividido conforme Quadro 1: 


\begin{tabular}{|c|c|c|}
\hline Classificação & Objetivo & Características \\
\hline Perfil do respondente & $\begin{array}{l}\text { Verificar a situação do } \\
\text { respondente no Programa } \\
\text { de Mestrado. }\end{array}$ & $\begin{array}{l}\text { - Condição (se é docente ou discente); } \\
\text { - Tempo de atuação; } \\
\text { - Programa ao qual está vinculado; }\end{array}$ \\
\hline Perfil interdisciplinar & $\begin{array}{c}\text { Verificar o perfil } \\
\text { interdisciplinar do } \\
\text { curso considerando as } \\
\text { disciplinas. }\end{array}$ & $\begin{array}{l}\text { - Percepção dos respondentes sobre o projeto } \\
\text { pedagógico do curso; }\end{array}$ \\
\hline $\begin{array}{c}\text { Importância } \\
\text { dos Elementos } \\
\text { promotores da } \\
\text { interdisciplinaridade }\end{array}$ & $\begin{array}{l}\text { Verificar a percepção } \\
\text { do respondente } \\
\text { sobre a presença de } \\
\text { interdisciplinaridade em } 5 \\
\text { aspectos. }\end{array}$ & $\begin{array}{l}\text { - Importância da interdisciplinaridade entre as } \\
\text { disciplinas; } \\
\text { - Integração entre as disciplinas e formação } \\
\text { profissional; } \\
\text { - Ausência de integração entre as disciplinas; } \\
\text { - Integração entre docentes e discentes; } \\
\text { - Promoção da interdisciplinaridade pela } \\
\text { coordenação. }\end{array}$ \\
\hline $\begin{array}{l}\text { Utilização de } \\
\text { mecanismos de } \\
\text { integração }\end{array}$ & $\begin{array}{c}\text { Identificar os mecanismos } \\
\text { utilizados para promoção } \\
\text { da integração entre as } \\
\text { disciplinas. }\end{array}$ & $\begin{array}{l}\text { - Leitura de textos e estudos de casos que contemplem } \\
\text { as duas áreas; } \\
\text { - Elaboração conjunta de artigos; } \\
\text { - Seminários, palestras e simpósios sobre temas de } \\
\text { interesse comum; } \\
\text { - Resolução conjunta de artigos; } \\
\text { Participação conjunta de professores em sala de } \\
\text { aula; } \\
\text { - Utilização conjunta de softwares de contabilidade; } \\
\text { - Utilização conjunta de softwares instrumentais; } \\
\text { Realização de estudos vinculados a núcleos de } \\
\text { pesquisa. }\end{array}$ \\
\hline $\begin{array}{l}\text { Metodologia do } \\
\text { ensino superior }\end{array}$ & $\begin{array}{l}\text { Verificar se o currículo do } \\
\text { curso oferece à disciplina } \\
\text { metodologia do ensino } \\
\text { superior. }\end{array}$ & - Verificar ausência ou presença. \\
\hline
\end{tabular}

Quadro 1: Instrumento de coleta de dados

A percepção dos discentes e docentes sobre interdisciplinaridade foi verificada por meio do grau de importância atribuído aos elementos promotores da interdisciplinaridade mediante 5 afirmações. Cada afirmação recebeu uma atribuição de grau de importância estabelecido por escala Likert de cinco pontos iniciando com discordo totalmente (1), discordo em grande parte (2), (3) indiferente, (4) discordo em grande parte e (5) discordo totalmente. Os questionários foram aplicados durante o período de 25 de setembro de 2010 a 22 de junho 2011. Para isso utilizou-se o software SurveyPRO, capaz de disponibilizar os questionários aos respondentes por meio eletrônico. Trata-se de um estudo exploratório e, portanto, não serão desenvolvidas hipóteses sobre possíveis resultados da inter-relação entre os elementos promotores da interdisciplinaridade estabelecidos.

Para análise dos resultados utilizou-se estatística descritiva e análise multivariada dos dados. Segundo Levine et al., a estatística descritiva tem o objetivo de coletar, sintetizar e apresentar dados, o que no presente estudo será demonstrado por meio das frequências e médias observadas. Para as questões que tratam do perfil do respondente, da interdisciplinaridade do curso e utilização de mecanismos de integração, será aplicada estatística descritiva. A análise multivariada permite a verificação simultânea de dados de uma amostra caracterizada por mais 
de duas variáveis correlacionadas (CORRAR et al., 2009).

Esta pesquisa analisa a presença de interdisciplinaridade nas disciplinas de contabilidade gerencial e de custos em programas de mestrado e não parte de uma relação de dependência entre os elementos promotores da interdisciplinaridade. Assim, aplica-se a técnica multivariada de interdependência denominada Análise Fatorial Exploratória (AFE) que não exige do pesquisador conhecimento prévio da relação de dependência entre variáveis (CORRAR et al., 2009). A AFE será aplicada para analisar os resultados das 5 afirmações do instrumento de pesquisa que tratam dos elementos promotores da interdisciplinaridade. Desse modo, busca-se com essa técnica, a exploração e simplificação das relações entre os elementos de promoção da interdisciplinaridade a partir da percepção de discentes e docentes.

Ressalta-se que Análise Fatorial Exploratória (AFE) é usada para o desenvolvimento de questionários que buscam medir habilidades ou características específicas de uma amostra (FIELD, 2009). Assim, a interpretação dos resultados será efetuada a partir dos parâmetros descritos no Quadro 2:

\begin{tabular}{|c|c|c|}
\hline Parâmetro & Objetivo do parâmetro & Demais características \\
\hline $\begin{array}{c}\text { Kaiser - Meyer - } \\
\text { Olkim. Medida de } \\
\text { adequação da Amostra } \\
\text { (MSA) }\end{array}$ & $\begin{array}{l}\text { Medir o quão adequado é a aplicação da } \\
\text { análise fatorial para explorar o problema de } \\
\text { pesquisa. A Estatística KMO varia entre } 0 \text { a } \\
\text { 1. (HAIR, 2005) }\end{array}$ & $\begin{array}{l}\text { Kaiser (1974, apud FIELD, 2009) } \\
\text { afirma que resultados abaixo de 0,5 } \\
\text { indicam inadequação da Análise } \\
\text { fatorial ao problema de pesquisa. }\end{array}$ \\
\hline $\begin{array}{l}\text { Teste de esfericidade de } \\
\text { Bartlet }\end{array}$ & $\begin{array}{l}\text { Indica se a relação entre as variáveis da } \\
\text { pesquisa são suficientes para aplicação da } \\
\text { análise fatorial. (CORRAR et al., 2009) }\end{array}$ & $\begin{array}{l}\text { Recomenda-se que o valor de Sig. Não } \\
\text { seja maior que } 0,05 \text {. }\end{array}$ \\
\hline $\begin{array}{l}\text { Total da variância } \\
\text { explicada }\end{array}$ & $\begin{array}{l}\text { Indicar o número de fatores que podem } \\
\text { explicar a variância dos componentes. } \\
\text { Na pesquisa os componentes iniciais são } \\
\text { identificados pelo número variáveis antes da } \\
\text { extração dos fatores. }\end{array}$ & $\begin{array}{l}\text { Não é estabelecido um parâmetro } \\
\text { máximo/mínimo de fatores e nem } \\
\text { um máximo/mínimo de variância } \\
\text { explicada. }\end{array}$ \\
\hline $\begin{array}{l}\text { Matriz de fatores } \\
\text { rotacionados }\end{array}$ & $\begin{array}{l}\text { Classificar de forma precisa as perguntas (ou } \\
\text { variáveis) de cada um dos fatores gerados } \\
\text { (CORRAR et al., 2009) }\end{array}$ & $\begin{array}{l}\text { Este resultado dependerá do numero de } \\
\text { variáveis (ou afirmações) de pesquisa. } \\
\text { Não há um número mínimo ou máximo } \\
\text { de fatores recomendados. }\end{array}$ \\
\hline Comunalidades & $\begin{array}{l}\text { Indicar quanto todos os fatores juntos são } \\
\text { capazes de explicar uma variável. }\end{array}$ & $\begin{array}{l}\text { Quão mais próximo de } 1 \text {, maior o poder } \\
\text { de explicação dos fatores. (CORRAR } \\
\text { et al., 2009) }\end{array}$ \\
\hline
\end{tabular}

Quadro 2: Parâmetros de avaliação da AFC

Fonte: adaptado de Hair et al. (2005), Field (2009) e Corrar et al. (2009)

Ainda que o parâmetro KMO de adequação da amostra tenha o objetivo de verificar a eficiência da aplicação da AFE ao problema de pesquisa, é possível obter uma medida de adequação da amostra individual para cada variável (afirmação). No que se refere ao total da variância explicada, os componentes desta pesquisa são representados pelas 5 variáveis: (1) Integração disciplinar; (2) Integração profissional; (3) Ausência de integração disciplinar; (4) Integração de docentes e discentes; (5) Estímulo à interdisciplinaridade. Desse modo, o método da análise fatorial exploratória será aplicado sobre as afirmações que verificaram a percepção dos respondentes no que se refere a importância dos elementos promotores da interdisciplinaridade nas disciplinas de contabilidade gerencial e de custos. No Quadro 3 
demonstra-se quais são os questionamentos e estabelece uma denominação para cada uma das variáveis.

\begin{tabular}{|c|c|c|}
\hline Variável & Afirmações & Identificação \\
\hline Integração disciplinar & $\begin{array}{l}\text { É de grande importância a integração das disciplinas de Custos } \\
\text { e Contabilidade Gerencial no processo de ensino-aprendizagem }\end{array}$ & $\mathrm{V}_{1}$ \\
\hline Integração profissional & $\begin{array}{l}\text { A integração entre as disciplinas de Custos e Contabilidade } \\
\text { Gerencial auxilia na melhor consolidação do conhecimento e } \\
\text { formação dos profissionais dedicados a gestão das organizações, } \\
\text { no exercício da docência e no desenvolvimento da pesquisa } \\
\text { acadêmica }\end{array}$ & $\mathrm{V}_{2}$ \\
\hline $\begin{array}{l}\text { Ausência de integração } \\
\text { disciplinar }\end{array}$ & $\begin{array}{l}\text { A ausência da integração entre as disciplinas de Custos e } \\
\text { Contabilidade Gerencial prejudica o projeto pedagógico do } \\
\text { curso }\end{array}$ & $\mathrm{V}_{3}$ \\
\hline $\begin{array}{l}\text { Integração de docentes e } \\
\text { discentes }\end{array}$ & $\begin{array}{l}\text { A interdisciplinaridade promove maior integração entre } \\
\text { discentes e docentes }\end{array}$ & $\mathrm{V}_{4}$ \\
\hline $\begin{array}{l}\text { Estímulo à } \\
\text { interdisciplinaridade }\end{array}$ & $\begin{array}{l}\text { O curso, através de sua coordenação, estimula atividades que } \\
\text { promovam a interdisciplinaridade }\end{array}$ & $\mathrm{V}_{5}$ \\
\hline
\end{tabular}

Quadro 3: variáveis de pesquisa

Fonte: os autores

Identificados como variáveis, as 5 afirmações representam os 5 componentes iniciais que poderão ser resumidos em quantidade menor de fatores. Adicionam-se à análise dos resultados da pesquisa dois problemas que podem surgir: 1) singularidade observada em pares de variáveis; e 2) correlações estatisticamente não significativas entre estas. A presença destes dois problemas pode ser verificada por meio da análise da matriz de correlação de Pearson (FIELD, 2009).

\section{DESCRIÇÃO E ANÁLISE DE DADOS}

\subsection{Estatísticas descritivas}

Nas Tabelas 2 e 3 apresentam-se a distribuição dos respondentes por instituição de ensino que compõe a amostra e o tempo de atuação profissional dos respondentes. Ressalte-se que a escala utilizada na Tabela 3 tem como base o estudo desenvolvido por Huberman (1989).

Tabela 2: Respondentes por instituição

\begin{tabular}{c|c|c}
\hline Instituições & Respondentes & \% \\
\hline 1 & 19 & 25 \\
\hline 2 & 10 & 13 \\
\hline 3 & 22 & 29 \\
\hline 4 & 20 & 26 \\
\hline 5 & 6 & 8 \\
\hline Total & 77 & 100 \\
\hline
\end{tabular}

Tabela 3: Experiência profissional

\begin{tabular}{c|c|c}
\hline Atuação profissional & Respondentes & $\mathbf{\%}$ \\
\hline De 1 até 3 anos & 32 & 42 \\
\hline De 4 até 6 anos & 11 & 14 \\
\hline De 7 até 25 anos & 30 & 39 \\
\hline De 26 até 35 anos & 2 & 26 \\
\hline De 36 até 40 anos & 2 & 26 \\
\hline Total & 77 & 100 \\
\hline
\end{tabular}

Apesar da distribuição de respondentes ser significativamente menor nas instituições 2 e 5 (13\% e $8 \%$, respectivamente), as respostas obtidas totalizam mais de $50 \%$ de discentes e docentes das instituições pesquisadas. A maioria dos respondentes da pesquisa (95\%) concentra- 
se em até 25 anos de experiência profissional na condição de docente, com participação relevante de respondentes com experiência entre 7 e 25 anos (39\%).

Ao referirem-se às suas percepções sobre a coordenação ou regimento do curso em contemplar a interdisciplinaridade nas disciplinas de contabilidade gerencial e de custos, $51 \%$ dos respondentes afirmam observar a presença de interdisciplinaridade no projeto pedagógico do curso; $6 \%$ mencionaram que não observam a interdisciplinaridade; e $43 \%$ desconhecem atitudes das coordenações em contemplar a interdisciplinaridade nos projetos pedagógicos.

$\mathrm{Na}$ Tabela 4 apresentam-se as médias de pontos obtidos para as afirmações que se referem à importância dos elementos promotores de interdisciplinaridade descritos no quadro 2 e 3. Das cinco afirmações, observa-se que em quatro delas, os resultados médios permaneceram no ponto 4 , indicando que docentes e discentes concordam em grande parte com a importância dos elementos promotores da interdisciplinaridade nas disciplinas de contabilidade gerencial e contabilidade de custos. A exceção ficou para a afirmação que buscou captar a percepção dos alunos em relação ao efeito sobre o projeto pedagógico dos programas em decorrência da ausência de interdisciplinaridade entre as disciplinas de Custos e Contabilidade Gerencial $\left(\mathrm{V}_{3}\right)$. Dos 77 respondentes 24 posicionaram-se de forma indiferente sobre esta afirmação.

Tabela 4: Média dos resultados da escala likert

\begin{tabular}{c|c|c|c|c|c|c}
\hline Instituição & Respondentes & \multicolumn{2}{c}{$\begin{array}{c}\text { V1 - Integração } \\
\text { disciplinar }\end{array}$} & \multicolumn{2}{c}{ V2 - Integração } \\
profissional & \multicolumn{2}{c}{$\begin{array}{c}\text { V3 - Ausência } \\
\text { de integração } \\
\text { disciplinar }\end{array}$} & $\begin{array}{c}\text { V4 - Integração } \\
\text { de docentes e } \\
\text { discentes }\end{array}$ & $\begin{array}{c}\text { V5 - Estímulo à } \\
\text { interdisciplinaridade }\end{array}$ \\
\hline 1 & 19,00 & 4 & 4 & 4 & 4 & 3 \\
\hline 2 & 10,00 & 4 & 4 & 3 & 4 & 4 \\
\hline 3 & 22,00 & 5 & 5 & 4 & 4 & 4 \\
\hline 4 & 20,00 & 5 & 4 & 3 & 4 & 4 \\
\hline 5 & 6,00 & 4 & 4 & 3 & 4 & 3 \\
\hline Total & 77,00 & 4,41 & 4,42 & 3,45 & 4,16 & 3,64 \\
\hline
\end{tabular}

Com o objetivo de verificar a percepção dos respondentes sobre os aspectos de interdisciplinaridade definidos na presente pesquisa, foi efetuado o agrupamento das frequências de respostas 1 e 2 e respostas 4 e 5 que indicam a concentração de respostas discordantes e concordantes, respectivamente. Ainda que esteja evidente maior frequência de concordâncias para as 5 afirmações aplicadas, observa-se na Tabela 5 concentração de respostas de indiferença sobre os questionamentos $\mathrm{V}_{3}, \mathrm{~V}_{4}$ e $\mathrm{V}_{5} \operatorname{com} 31,17 \%, 22,08 \%$ e $33,77 \%$, respectivamente.

O conteúdo das afirmações $\mathrm{V}_{3}, \mathrm{~V}_{4}$ e $\mathrm{V}_{5}$ está associado às ações de planejamento e execução de medidas que estimulem a interdisciplinaridade entre as duas disciplinas. Estas afirmações buscam verificar a percepção do docente e discente sobre possíveis prejuízos causados pela ausência de integração entre as duas disciplinas, sobre a promoção de integração entre docentes e discentes e de estímulo à interdisciplinaridade.

A constatação desta indiferença sobre a importância destes elementos pode indicar uma lacuna de ações e procedimentos necessários para contemplar a interdisciplinaridade entre as 
disciplinas.

Tabela 5: Frequência de respostas para cada variável

\begin{tabular}{c|ccccc}
\hline Respostas & $\begin{array}{c}\text { V1 - } \\
\text { Integração } \\
\text { disciplinar }\end{array}$ & $\begin{array}{c}\text { V2 - } \\
\text { Integração } \\
\text { profissional }\end{array}$ & $\begin{array}{c}\text { V3 - Ausência } \\
\text { de integração } \\
\text { disciplinar }\end{array}$ & $\begin{array}{c}\text { V4- } \\
\text { Integração } \\
\text { de docentes e } \\
\text { discentes }\end{array}$ & $\begin{array}{c}\text { V5 - Estímulo à } \\
\text { interdisciplinaridade }\end{array}$ \\
\hline $\begin{array}{c}\text { Discordam em } \\
\text { grande parte e } \\
\text { totalmente }\end{array}$ & $2,60 \%$ & $0,00 \%$ & $20,78 \%$ & $6,49 \%$ & $16,88 \%$ \\
\hline Indiferente & $3,90 \%$ & $10,39 \%$ & $31,17 \%$ & $22,08 \%$ & $33,77 \%$ \\
\hline $\begin{array}{c}\text { Concordam em } \\
\text { grande parte e } \\
\text { totalmente }\end{array}$ & $93,51 \%$ & $89,61 \%$ & $48,05 \%$ & $71,43 \%$ & $49,35 \%$ \\
\hline
\end{tabular}

Em complemento à Tabela 5, a Tabela 6 descreve não haver diferenças relevantes de percepção de docentes e discentes sobre a importância dos elementos promotores da interdisciplinaridade. Para as 5 afirmações, as percepções de docentes e discentes não chegam a se diferenciar em um ponto.

Tabela 6: percepção de docentes e discentes

\begin{tabular}{c|ccccc}
\hline Respostas & $\begin{array}{c}\text { V1 - } \\
\text { Integração } \\
\text { disciplinar }\end{array}$ & $\begin{array}{c}\text { V2 - } \\
\text { Integração } \\
\text { profissional }\end{array}$ & $\begin{array}{c}\text { V3 - Ausência } \\
\text { de integração } \\
\text { disciplinar }\end{array}$ & $\begin{array}{c}\text { V4 - } \\
\text { Integração } \\
\text { de docentes e } \\
\text { discentes }\end{array}$ & $\begin{array}{c}\text { V5 - Estímulo à } \\
\text { interdisciplinaridade }\end{array}$ \\
\hline Docentes & 4,4 & 4,6 & 3,3 & 4,4 & 3,6 \\
Discentes & 4,5 & 4,5 & 3,5 & 4,0 & 3,6 \\
\hline
\end{tabular}

Por meio da Tabela 7 verificam-se quais os mecanismos utilizados pelas instituições para promoção da integração das disciplinas de contabilidade gerencial e de custos. Segundo os respondentes, destacam-se três mecanismos: leitura de textos e estudos de casos que contemplam as duas áreas, elaboração de artigos e seminários.

Tabela 7: Mecanismos de integração utilizados pelos programas

\begin{tabular}{l|c|c}
\hline \multicolumn{1}{c}{ Mecanismos de integração } & Quant. & $\%$ \\
\hline Leitura de textos e estudos de casos que contemplem as duas áreas & 51 & $27,27 \%$ \\
\hline Elaboração conjunta de artigos & 42 & $22,46 \%$ \\
\hline Seminários, palestras e simpósios sobre temas de interesse comum & 35 & $18,72 \%$ \\
\hline Realização de pesquisa em grupos de pesquisa & 24 & $12,83 \%$ \\
\hline Participação conjunta de professores em sala de aula & 12 & $6,42 \%$ \\
\hline Resolução conjunta de exercícios & 8 & $4,28 \%$ \\
\hline Participação conjunta de softwares de contabilidade & 8 & $4,28 \%$ \\
\hline Participação conjunta de softwares instrumentais & 7 & $3,74 \%$ \\
\hline
\end{tabular}

Os resultados indicam que atividades relacionadas à discussão de textos e estudos de caso, e a realização de eventos envolvendo temas relacionados, são os mecanismos mais utilizados pelos programas de mestrado para promover a integração entre as duas disciplinas. 


\subsection{Inter-relação das variáveis de pesquisa}

Na Tabela 8 apresenta-se a matriz de correlação de Pearson para as variáveis descritas no quadro 3. Há correlação significativa entre a maioria dos pares de variáveis. Além disso, não foi observada a presença de correlações acima de 0,9 , o que indicaria a presença do problema de singularidade (seção). Uma das condições de aplicabilidade da AFE consiste na associação entre as variáveis de pesquisa, ainda que esta relação não seja perfeita (FIELD, 2009). O atendimento desta condição pode ser verificado, já que a força de associação das variáveis de interdisciplinaridade medidas por meio dos coeficientes de correlação é estatisticamente significativa em 16 das 20 combinações possíveis.

Tabela 8: Correlação entre as varáveis

\begin{tabular}{|c|c|c|c|c|c|}
\hline & $\begin{array}{l}\mathrm{V}_{1} \text { - Integração } \\
\text { disciplinar }\end{array}$ & $\begin{array}{c}\mathrm{V}_{2}-\text { Integração } \\
\text { profissional }\end{array}$ & $\begin{array}{c}V_{3}-\text { Ausência } \\
\text { de integração } \\
\text { disciplinar }\end{array}$ & $\begin{array}{c}\mathrm{V}_{4} \text { - Integração } \\
\text { de docentes e } \\
\text { discentes }\end{array}$ & $\begin{array}{c}\mathbf{V}_{5} \text { - Estímulo à } \\
\text { interdisciplinaridade }\end{array}$ \\
\hline $\begin{array}{l}\text { V1 - Integração } \\
\text { disciplinar }\end{array}$ & 1 &, $603^{* *}$ &, $306^{* *}$ &, $227^{*}$ & 148 \\
\hline p-value & - &, 000 &, 007 &, 047 & $\underline{198}$ \\
\hline $\begin{array}{l}\text { V2 - Integração } \\
\text { profissional }\end{array}$ &, $603^{* *}$ & 1 &, $459^{* *}$ &, $335^{* *}$ &, $339^{* *}$ \\
\hline p-value &, 000 & - &, 000 &, 003 &, 003 \\
\hline $\begin{array}{c}\text { V3 - Ausência de } \\
\text { integração disciplinar }\end{array}$ &, $306^{* *}$ &, $459^{* *}$ & 1 &, $361^{* *}$ &, 113 \\
\hline p-value & $\underline{007}$ & $\underline{000}$ & - & $\underline{001}$ & $\underline{328}$ \\
\hline $\begin{array}{l}\text { V4 - Integração de } \\
\text { docentes e discentes }\end{array}$ &, $227^{*}$ & $335^{* *}$ &, $361^{* *}$ & 1 &, $397^{* *}$ \\
\hline p-value &, 047 &, 003 &, 001 & - &, 000 \\
\hline $\begin{array}{l}\text { V5 - Estímulo à } \\
\text { interdisciplinaridade }\end{array}$ &, 148 &, $339^{* *}$ &, 113 &, $397^{* *}$ & 1 \\
\hline p-value & $\underline{.198}$ &, 003 &, 328 & $\underline{000}$ & - \\
\hline
\end{tabular}

As medidas apresentadas na Tabela 9 demonstram os parâmetros de adequação para uso da AFE. A estatística KMO apresentou o resultado de 0,644 viabilizando a utilização da AFE. Adicionalmente, o teste de esfericidade mostra a existência de relação estatisticamente significativa entre as variáveis ao nível de $5 \%$.

Tabela 9: Adequação da amostra

\begin{tabular}{cr}
\hline Medidas & \multicolumn{1}{c}{ Parâmetros } \\
\hline KMO - Medida de adequação da amostra & 0,644 \\
Teste de esfericidade & 83,165 \\
\hline$p$-Value & 0,000 \\
\hline
\end{tabular}

Por meio da diagonal principal da tabela 10 é apresentado o resultado da estatística KMO para cada uma das variáveis de pesquisa, onde todas obtiveram escores acima de 0,500. 
Tabela 10: Matriz anti-imagem

\begin{tabular}{|c|c|c|c|c|c|}
\hline & $\begin{array}{l}\mathrm{V}_{1} \text { - Integração } \\
\text { disciplinar }\end{array}$ & $\begin{array}{c}\mathrm{V}_{2}-\text { Integração } \\
\text { profissional }\end{array}$ & $\begin{array}{c}\mathrm{V}_{3} \text { - Ausência } \\
\text { de integração } \\
\text { disciplinar }\end{array}$ & $\begin{array}{c}\mathrm{V}_{4} \text { - Integração } \\
\text { de docentes e } \\
\text { discentes }\end{array}$ & $\begin{array}{c}V_{5}-\text { Estímulo à } \\
\text { interdisciplinaridade }\end{array}$ \\
\hline $\begin{array}{l}V_{1} \text { - Integração } \\
\text { disciplinar }\end{array}$ &, $640^{\mathrm{a}}$ &,- 537 &,- 021 &,- 053 & ,086 \\
\hline $\begin{array}{l}V_{2}-\text { Integração } \\
\text { profissional }\end{array}$ &,- 537 &, $627^{a}$ &,- 330 &,- 048 &,- 276 \\
\hline $\begin{array}{l}V_{3}-\text { Ausência de } \\
\text { integração disciplinar }\end{array}$ &,- 021 &,- 330 &, $685^{a}$ &,- 278 & ,140 \\
\hline $\begin{array}{l}V_{4} \text { - Integração de } \\
\text { docentes e discentes }\end{array}$ &,- 053 &,- 048 &,- 278 &, $691^{\mathrm{a}}$ &,- 346 \\
\hline $\begin{array}{l}\mathbf{V}_{5} \text { - Estímulo à } \\
\text { interdisciplinaridade }\end{array}$ & ,086 &,- 276 & ,140 &,- 346 &, $579^{a}$ \\
\hline
\end{tabular}

A Tabela 10 complementa os resultados da Tabela 9 na medida em que demonstra que além da amostra ser adequada para análise de fatorial, não será necessária a exclusão de nenhuma das variáveis de pesquisa, já que todas ficaram com a estatística KMO individual acima do mínimo recomendado.

Tabela 11: Total da variância explicada

\begin{tabular}{c|c|c|c|c|c|c}
\hline \multirow{2}{*}{ Componentes } & \multicolumn{3}{|c|}{ Autovalores iniciais } & \multicolumn{3}{c}{ Extração da soma das cargas } \\
\cline { 2 - 7 } & Total & $\%$ de Variância & Acumulado \% & Total & \% da Variância & Acumulado \% \\
\hline 1 & $\mathbf{2 , 3 4 9}$ & $\mathbf{4 6 , 9 7 2}$ & $\mathbf{4 6 , 9 7 2}$ & $\mathbf{2 , 3 4 9}$ & $\mathbf{4 6 , 9 7 2}$ & $\mathbf{4 6 , 9 7 2}$ \\
\hline 2 & $\mathbf{1 , 0 1 2}$ & $\mathbf{2 0 , 2 3 5}$ & $\mathbf{6 7 , 2 0 7}$ & $\mathbf{1 , 0 1 2}$ & $\mathbf{2 0 , 2 3 5}$ & $\mathbf{6 7 , 2 0 7}$ \\
\hline 3 &, 783 & 15,660 & 82,867 & & & \\
\hline 4 &, 529 & 10,576 & 93,443 & & & \\
\hline
\end{tabular}

A AFE por meio da Tabela 11 indica que dos 5 componentes iniciais da pesquisa representados pelas variáveis $\mathrm{V}_{1}, \mathrm{~V}_{2}, \mathrm{~V}_{3}, \mathrm{~V}_{4}$ e $\mathrm{V}_{5}, 67 \%$ do total da variância observada pelos componentes originais pode ser explicado por dois fatores, sendo 45,67\% explicado pelo primeiro e $20,31 \%$ pelo segundo fator.

As variáveis que compõem os dois fatores podem ser observadas na tabela 12 que apresenta a matriz de componentes rotacionados. O primeiro fator é composto pelas variáveis de Integração Disciplinar, Integração Profissional e Ausência de integração. O segundo é composto pelas variáveis de Integração entre docentes e discentes e estímulo a interdisciplinaridade. Deve-se então observar o conteúdo das perguntas e buscar semelhanças temáticas entre elas. Neste sentido, Field (2009, p. 558) afirma que "se o fator matemático produzido pela análise fatorial representa um constructo real, temas comuns entre perguntas com cargas altas podem nos ajudar a identificar o que o constructo pode ser". 
Tabela 12: Matriz de fatores rotacionados

\begin{tabular}{l|c|c}
\hline \multicolumn{1}{c|}{ Variáveis } & \multicolumn{2}{c}{ Componentes } \\
\cline { 2 - 3 } & $\mathbf{1}$ & $\mathbf{2}$ \\
\hline $\mathrm{V}_{1}$ - Integração disciplinar &, 834 & \\
\hline $\mathrm{V}_{2}$ - Integração profissional &, 817 & \\
\hline $\mathrm{V}_{3}$ - Ausência de integração disciplinar &, 685 &, 874 \\
\hline $\mathrm{V}_{4}$ - Integração de docentes e discentes & &, 752 \\
\hline $\mathrm{V}_{5}$ - Estímulo à interdisciplinaridade & & 20,23 \\
\hline Total da Variância explicada & 46,97 & \\
\hline
\end{tabular}

As perguntas do fator 1 estão associadas: a) à integração das disciplinas de contabilidade gerencial e de custos no processo de ensino-aprendizagem; b) ao auxílio ao desenvolvimento profissional dos respondentes; e c) ao efeito da ausência desta integração sobre a promoção da interdisciplinaridade nos programas de mestrado em contabilidade. A relação destas três variáveis sugere significativa influência da integração entre as disciplinas para promoção da interdisciplinaridade. Como objetivo da análise fatorial exploratória é buscar a formação de constructos a partir das cargas das variáveis de pesquisa agrupadas em fatores, verifica-se que o conteúdo comum das perguntas refere-se à integração das disciplinas contabilidade gerencial e de custos. Assim, pode-se denominar o fator 1 com um constructo de "Integração Acadêmica e Profissional".

As perguntas do fator 2 referem-se à integração de alunos e professores e ao estímulo de atividades que promovam a interdisciplinaridade por parte da coordenação dos cursos de mestrado pesquisados. O agrupamento destas variáveis no fator 2 , conforme demonstra a tabela 8, associa-os aos aspectos relacionados a uma postura interdisciplinar por parte de alunos, professores e por meio da coordenação. Nesta direção, pode-se denominar o fator 2 como constructo de "Postura Interdisciplinar". As comunalidades representam a proporção da variância comum dentro de cada variável e sua análise permite verificar o quanto da variância é efetivamente comum depois dos fatores já terem sido extraídos e apresentados na Tabela 12 (FIELD, 2009).

Tabela 13: comunalidades das variáveis

\begin{tabular}{l|c}
\hline \multicolumn{1}{c}{ Variáveis } & Extração \\
\hline $\mathrm{V}_{1}$ - Integração disciplinar & 0,697 \\
\hline $\mathrm{V}_{2}$ - Integração profissional & 0,751 \\
\hline $\mathrm{V}_{3}$ - Ausência de integração disciplinar & 0,501 \\
\hline $\mathrm{V}_{4}$ - Integração de docentes e discentes & 0,645 \\
\hline $\mathrm{V}_{5}$ - Estímulo à interdisciplinaridade & 0,767 \\
\hline \multicolumn{2}{c}{ Média } \\
\hline
\end{tabular}

A Tabela 13 indica que 69,7\% da variância associada à variável que se refere à integração disciplinar, tem a variância compartilhada com as outras 4 variáveis. Segundo Corrar et al. 
(2009), quanto mais próximo de 1, maior o poder de explicação dos fatores extraídos pela análise fatorial. A variável que compartilha maior proporção de sua variância é aquela que se refere ao estímulo, por parte da coordenação dos cursos, de atividades que promovam a interdisciplinaridade das disciplinas de contabilidade gerencial e de custos $(0,767)$. A variável que compartilha menor proporção de variância refere-se ao efeito da ausência de integração das disciplinas com o projeto pedagógico do mestrado $(0,501)$.

\section{CONSIDERAÇÕES FINAIS}

A presente pesquisa teve como objetivo verificar a percepção de docentes e discentes sobre a prática de interdisciplinaridade nas disciplinas de contabilidade gerencial e contabilidade de custos, ministradas nos cursos de mestrado de 5 instituições de ensino brasileiras. A pesquisa contou com 77 respondentes, sendo 67 discentes e 10 docentes de programas de mestrado e foi realizada no período de 25/09/2010 a 25/06/2011.

Sobre a percepção de presença de interdisciplinaridade nos projetos pedagógicos dos programas de mestrado pesquisados, os resultados da pesquisa indicam não ser possível afirmar que a maioria relevante dos respondentes observa a presença de práticas de interdisciplinaridade. A pesquisa demonstra que $43 \%$ dos respondentes afirmaram desconhecer e $6 \%$ afirmaram não haver nos programas de mestrado ações ou medidas que contemplem a prática de interdisciplinaridade. Estes resultados realçam a importância da criação e implementação de mecanismos que limitem o isolamento das disciplinas de contabilidade gerencial e de custos em programas de mestrado e que reforcem a necessidade de articulação de padrões curriculares para a melhor compreensão da complexa realidade empresarial (MANECHINE, 2006).

Apesar dos diferentes instrumentos de pesquisa utilizados e dos diferentes públicos de respondentes, os resultados do presente trabalho apresentam semelhanças aos de Padoan e Clemente (2006), que evidenciaram a baixa importância atribuída a disciplinas de extenso escopo teórico, segundo a percepção de docentes, em 8 instituições de ensino superior.

Ao que se refere à importância dos elementos promotores de interdisciplinaridade definidos neste trabalho, os resultados mostram que a percepção de discentes e docentes pesquisados é semelhante. Apesar de quase metade (49\%) dos respondentes observarem a ausência de práticas de interdisciplinaridade nos projetos pedagógicos dos programas, o resultado foi de reconhecimento da importância dos elementos promotores de interdisciplinaridade nas disciplinas de contabilidade gerencial e de custos. Este reconhecimento sobre a importância dos elementos promotores de interdisciplinaridade, aliado à fraca concordância (51\%) sobre a presença de interdisciplinaridade nos projetos pedagógicos, sugere haver a apreciação isolada do conteúdo destas duas disciplinas conforme reforça Machado (1995).

Por meio da aplicação da técnica da Análise Fatorial Exploratória (AFE) foi possível desenvolver dois constructos capazes de agrupar, com consistência teórica, temáticas relacionadas sobre a importância dos elementos promotores de interdisciplinaridade na percepção de docentes e discentes. Estes constructos foram identificados como "Integração Acadêmica" e "Postura Interdisciplinar".

Como sugestão para pesquisas futuras, recomenda-se desenvolver instrumentos de 
pesquisa que considerem uma quantidade mais elevada de variáveis que buscam captar a percepção de docentes e discentes sobre a prática de interdisciplinaridade. Em consequência, maior número de variáveis estaria presente em dois os mais constructos indicados pela técnica da AFE, cuja consistência teórica e prática poderiam demonstrar com mais abrangência a percepção de docentes e discentes sobre os elementos promotores da interdisciplinaridade em programas de mestrado.

\section{REFERÊNCIAS}

ALTHOFF, N. S.; DOMINGUES, M. J. C. S. Práticas interdisciplinares nos cursos de graduação em Ciências Contábeis: mito ou realidade. In: CONGRESSO DAASSOCIAÇÃO NACIONAL DOS PROGRAMAS DE PÓS-GRADUAÇÃO EM CONTABILIDADE, 2, 2008, Salvador. Anais... Salvador: ANPCONT, 2008, p. 1-16.

ALTHEMAN. E. A interdisciplinaridade no ensino superior de administração de empresas: possibilidades e dificuldades de efetivação. In: SEMEAD, 3, 1998, São Paulo. Seminários em Administração. São Paulo: Programa de Pós-Graduação em Administração - FEA/USP, 1998. Disponível em: $<$ http://www.ead.fea.usp.br/Semead/3semead/pdf/Ensino/Art118.pdf $>$ Acesso em: 10 dez. 2010.

BERNARDO, D. C. R.; NASCIMENTO, J. P. B.; NAZARETH, L. G. C. Representações do Ensino, Pesquisa e Interdisciplinaridade dos Cursos de Ciências Contábeis no Estado de Minas Gerais. Revista de contabilidade Vista e Revista, n. 3, p. 111-133, jul./set. 2010.

CORRAR, L. J.; PAULO, E.; DIAS FILHO, J. M.; RODRIGUES, A. Análise multivariada para os cursos de administração, ciências contábeis e economia. Fundação Instituto de Pesquisas Contábeis, Atuariais e Financeiras - FIPECAFI. São Paulo: Atlas, 2009.

DALBEN, A. I. L. F.; CASTRO, E. V. A relação pedagógica no processo escolar: sentidos e significados. In: TEIXEIRA, A. B. M. Temas atuais em didática. Belo Horizonte: Editora UFMG, 2010. p. 13-61.

FAZENDA, I. C. A. A Integração e Interdisciplinaridade no Ensino Brasileiro. 2. ed. São Paulo: Loyola, 1992. 107p.

FERREIRA, L. S. Introdução a noção de interdisciplinaridade. In: Prática Intedisciplinares na Escola. São Paulo: Cortez, 2001. p. 33-35.

FIELD, A. Descobrindo estatística utilizando o SPSS. Tradução Lorí Viali. 2. ed. Porto Alegre: Artmed, 2009. 688p.

FRAGOSO, A. R.; RIBEIRO FILHO, J. F.; LIBONATI, J. J. Um estudo aplicado sobre o impacto da interdisciplinaridade no processo de pesquisa dos doutores em contabilidade no Brasil. Revista Universo Contábil. Blumenau, v. 2, n. 1, p. 103-112, jan./abr., 2006.

GERMAIN, C. Interdisciplinarité et Globalité: Remarques dordre Épistèmologique. Revue des Sciences del Éducation. XVII, p. 142-152. 1991. 
GUSDORF, G. Réflexions sur l'interdisciplinarité. Bulletin de Psychologie. XLIII, 397, p. 847-868. 1990.

HAIR, J.F. et al. Análise multivariada de dados. 5. ed. Porto Alegre: Bookman, 2005. 593p.

HUBERMAN, M. Les cycles de vie des enseignants. Neuchatel:Delachaux et Nestlè, 1989.

KAISER, H. F. An Index of Factorial Simplicity. Psychometrics, 1974.

KRASILCHIK, M. Planejamento educacional: estruturando o currículo. In: Marcondes, E; GONÇALVES, E. L. Educação Médica. São Paulo: Sarvier, 1998. p. 5-9.

LAFFIN, M. Projeto político-pedagógico nos cursos de ciências contábeis. Revista Brasileira de Contabilidade. v. 33, n. 3, p. 85-97, São Paulo, jul./ago., 2004.

LEONE, G. S. G. Contabilidade de Custos. 2 ed. São Paulo: Atlas, 2000.

LEVINE, D. M. et al. Estatística: Teoria e aplicações. Tradução Teresa Cristina Padilha de Souza. Rio de Janeiro: LTC, 2008. 752p.

LUCK, H. Pedagogia Interdisciplinar: Fundamentos Teórico-Metodológicos. Petrópolis: Editora Vozes, 1995.

MACHADO, N. J. Epistemologia e didática: as concepções de conhecimento e inteligência e a prática docente. São Paulo: Cortez, 1995. 304p.

MANECHINE, S. R. S. Construção de signos matemáticos: uma proposta didáticometodológica para as séries iniciais do ensino fundamental. 2006. 307f. Tese (Doutorado em Educação). Programa de Doutorado em Educação para Ciências - Faculdade de Ciências da Universidade Estadual Paulista Júlio de Mesquita Filho, Bauru, 2006.

MARTINS, E. Contabilidade de Custos. 9. ed. São Paulo: Atlas, 2008. 378p.

MAUSS, C. V.; SOUZA, M. A. Gestão de Custos aplicada ao setor público. São Paulo: Atlas, 2008.

MORAES, V. F. J.; ARAÚJO, A. O.; ARAÚJO, M. I. O. Percepção sobre a atitude interdisciplinar dos professores no curso de ciências contábeis: um estudo nas universidades Norte RioGrandenses. Revista de Contabilidade e Organizações, n. 7, p. 127-144, set./dez. 2009.

MORIN, E. A religação dos saberes. 3. ed. Rio de Janeiro: Bertrand Brasil, 2002. 588p.

MORIN, E. Ciência com consciência. Rio de Janeiro: Bertrand Brasil, 1999. 350p.

NOGUEIRA, N. R. Interdisciplinaridade aplicada. 3. ed. São Paulo: Érica, 1998. 120p.

PADOAN, F. A. C; CLEMENTE, A. A interdisciplinaridade no ensino da contabilidade - um estudo empírico da percepção dos docentes. In: CONGRESSO USP DE CONTROLADORIA E CONTABILIDADE, n. 06, 2006, São Paulo. Anais... São Paulo. Disponível em: $<$ http:// www.congressousp.fipecafi.org/artigos62006/551.pdf> Acesso em: 15 nov. 2010.

PADOVEZE, C. L. Contabilidade Gerencial. 3. ed. São Paulo: Atlas, 2000. 432p. 
PASSOS, I. C. A interdisciplinaridade no ensino e na pesquisa da contabilidade: um estudo empírico do município de São Paulo. 2004. 164f. Dissertação (mestrado). Faculdade de Economia, Administração e Contabilidade. Universidade de São Paulo. São Paulo, 2004.

PIAGET, J. Epistemologie des rélations interdisciplinaires. In: Ceri (eds.) L'interdisciplinarité: problèmes d'enseignement et de recherche dans les universités. 131-144. Paris: UNESCO/ OCDE. 1972.

ROSLENDER, R.; DILLARD, J. Reflections on the interdisciplinary perspectives on accounting research. Critical Perspectives on Accounting, n. 14, v. 3, p. 325-351, 2003.

SANTOMÉ, J. T. Globalização e interdisciplinaridade: o currículo integrado. Porto Alegre: Artes Médicas, 1998. 278p.

WALKER, S. P. Accounting in history. Accounting Historians Journal, v. 33 n. 2, p. 233-59. 2005.

ZABALA, A. Enfoque globalizador e pensamento complexo: uma proposta para o currículo escolar. Porto Alegre: Artmed, 2002. 248p. 


\section{Endereço dos Autores:}

\section{Claudio Marcelo Edwards Barros}

Universidade Federal do Paraná

Rua Antônio Ricardo Dos Santos, 452

Bairro Hauer - Curitiba, PR - Brasil

81.610-160

\section{Luiz Carlos Lemos Júnior}

Pontifícia Universidade Católica de Campinas

Rodovia D. Pedro I, KM 136

Parque das Universidades - Campinas, SP - Brasil

$13.086-900$

\section{Romualdo Douglas Colauto}

Universidade Federal do Paraná

Av. Prefeito Lothário Meissner, 632 - Campus III

Jardim Botânico - Curitiba, PR - Brasil

80210-170

\section{Simone Bernardes Voese}

Universidade Federal do Paraná

Av. Prefeito Lothário Meissner, 632 - Campus III

Jardim Botânico - Curitiba, PR - Brasil

80210-170 